Originalien

Z Gerontol Geriat 2019 · 52:743-750 https://doi.org/10.1007/s00391-019-01645-w Eingegangen: 15. Januar 2019

Angenommen: 15. September 2019

Online publiziert: 6. November 2019

(c) Der/die Autor(en) 2019

\section{Hinführung zum Thema}

„Moral distress" (MD) wird mit reduzierter Arbeitszufriedenheit und der Absicht des Berufsausstiegs in Verbindung gebracht. Obwohl anzunehmen ist, dass einige Besonderheiten des Settings Pflegeheim die Entstehung von MD begünstigen, wurde das Phänomen bislang primär im Akutbereich untersucht. Dabei wird zumeist auf eine enge Definition rekurriert, die MD auf Situationen reduziert, in denen die Pflegekraft am moralisch korrekten Handeln gehindert wird. $\mathrm{Zu}$ nehmend wird die Forderung laut, auch moralische Unsicherheit unter MD zu subsumieren, um das Phänomen in seiner Gesamtheit abzubilden. In der vorliegenden Studie wird exploriert, ob und in welcher Form Pflegekräfte im Pflegeheim MD erleben.

\section{Hintergrund und Fragestellung}

Das Setting Pflegeheim weist einige Besonderheiten auf, die im Hinblick auf die Entstehung von MD von Bedeutung sind [8]. Dazu zählen insbesondere die fragmentarische ärztliche Präsenz, wodurch beispielsweise Entscheidungen für oder gegen einen Krankenhaustransport oftmals vom Pflegepersonal getroffen werden müssen $[9,13]$, die häufige Konfrontation mit Tod und Sterben sowie die

Hinweis zum geschlechtersensiblen Sprachgebrauch: Aus Gründen der besseren Lesbarkeit wird im Text nur die männliche Form verwendet. Gemeint ist stets sowohl die weibliche als auch die männliche Form.

\author{
Olivia Kada' ${ }^{1}$ Tanja Lesnik ${ }^{2}$ \\ 'Studiengang Gesundheits- und Pflegemanagement, FH Kärnten, Feldkirchen i.K.,Österreich \\ ${ }^{2}$ Klinikum Klagenfurt am Wörthersee, Studiengang Gesundheits- und Pflegemanagement, FH Kärnten, \\ Kärnten, Österreich
}

\title{
Facetten von „moral distress" im Pflegeheim
}

\section{Eine qualitative Studie mit examinierten Pflegekräften}

besonders engen Beziehungen zwischen Pflegepersonal und Bewohnern [24]. "Moral distress" im Pflegeheim resultiert oftmals aus der gefühlten Machtlosigkeit, die entsteht, wenn die Vorstellungen eines würdevollen Sterbens zwischen Pflegekraft und Angehörigen, Ärzten oder Kollegen auseinanderklaffen [26].

Dennoch wurde MD bislang insbesondere im Notfall- und Intensivbereich untersucht [14, 19, 27], es gibt jedoch Hinweise auf eine vergleichsweise stärkere Ausprägung von MD im Pflegeheim [2]. In seiner ursprünglichen Definition (Jameton, 1984 zit. n. [7]) bezeichnet MD die Stressreaktion, die entsteht, wenn eine Pflegeperson die moralisch richtige Handlungsweise identifiziert hat, durch externe oder interne Barrieren jedoch an der Umsetzung gehindert wird $[4,18]$. Der Großteil der theoretischen Modelle, Studien und Messinstrumente (z.B. [1, $12,20,21])$ rekurriert auf diese enge Definition [8], die auch als ,constraint MD“ (cMD) bezeichnet wird [5]. Dabei wird postuliert, dass cMD negative Auswirkungen auf die Pflegeperson (z. B. Resignation und Rückzug) und somit auch für die Bewohner und die Institution (z. B. reduzierte Patientenzufriedenheit, hohe Personalfluktuation) hat [1]. Die zentrale Rolle institutioneller Rahmenbedingungen, wie etwa unzureichender interdisziplinärer Kooperation, für die Entstehung von cMD wird im "Moral-crescendo“Modell betont [4]. Einige Autoren, insbesondere Fourie [5], plädieren jedoch für eine breitere Definition, die neben cMD auch Stressreaktionen in Situatio- nen moralischer Unsicherheit, Dilemmata und Konflikte umfasst (,uncertainty MD“, uMD; auch [18]). Diese breitere Konzeption erscheint auch vor dem Hintergrund empirischer Befunde sinnvoll (z. B. $[9,11,13])$ und dürfte insbesondere im Pflegeheim bedeutsam sein. Preshaw et al. [22] konstatieren einen generellen Mangel an Studien zu ethischen Herausforderungen im Pflegeheim - insbesondere im deutschsprachigen Raum [27] - sowie eine uneinheitliche begriffliche Verwendung. Qualitative und quantitative Studien im Pflegeheimsetting, die explizit auf das Konzept MD Bezug nehmen, rekurrieren auf die enge Definition von MD [20, 21, 24, 26] und sind teilweise thematisch begrenzt, etwa auf die Pflege von Menschen mit Demenz [21, 24].

Ziele der vorliegenden Studie waren die Exploration und Beschreibung der Facetten von MD im Kontext Pflegeheim. Dabei sollten folgende Fragen beantwortet werden:

1. Wie stark wird moralischer Stress im Kontext Pflegeheim erlebt? Der Ausprägungsgrad wird dabei über Frequenz und Intensität des Erlebens von $\mathrm{MD}[1,12,21]$ operationalisiert, wobei in der vorliegenden Studie neben $\mathrm{cMD}$ auch uMD berücksichtigt wird.

2. In welcher Form zeigt sich MD im Kontext Pflegeheim? Die Diskussion um die Definition von MD (v. a. $[5,18])$ aufgreifend, werden Erfahrungen von MD durch Barrieren 
(cMD) sowie auch aufgrund von Unsicherheit (uMD) exploriert.

3. In welchen Situationen wird MD erlebt? In welchen Situationen wird welche Form von MD (cMD vs. uMD) beschrieben?

4. Welche externen und internen Barrieren verhindern das moralisch korrekte Handeln und führen somit zu cMD?

5. Was sind die Quellen bzw. Ursachen von moralischem Stress durch Unsicherheit (uMD).

6. Welche Ressourcen zur Vermeidung und Bewältigung von MD sind bereits vorhanden, und welche werden als potenziell hilfreich erachtet?

\section{Methode}

\section{Stichprobe}

Zunächst erfolgte die Auswahl von 5 Pflegeheimen mittels eines qualitativen Stichprobenplans [3,23], wobei als Kriterien die Trägerorganisation $(n=3)$ und die geografische Lage ( $n=4$ Bezirke) festlegt wurden. Über die Heime hinweg wurde eine annähernde Gleichverteilung der Anzahl der Probanden angestrebt. Auf diese Weise sollte eine heterogene Stichprobe erreicht werden, um etwaige $\mathrm{Be}$ sonderheiten eines einzelnen Heims bzw. Trägers auszugleichen. Die Pflegedienstleitungen der betreffenden Heime wurden per E-Mail über das Ziel der Studie informiert und ersucht, den Forscherinnen Kontakt zu je ca. 4 Pflegepersonen herzustellen, die zu einem Interview bereit wären. Aufdiese Weise konnten 21 diplomierte (examinierte) Pflegepersonen aus 5 Pflegeheimen in Kärnten (Österreich) im Alter zwischen 23 und 57 Jahren $(\mathrm{Md}=38)$ mit einer Berufserfahrung zwischen 1,5 und 20 Jahren $(\mathrm{Md}=8,5)$ für die Teilnahme an der Studie gewonnen werden. Bis auf eine Person waren die Studienteilnehmer weiblich. Die durchschnittliche Interviewdauer betrug $24 \mathrm{~min}$. Saturierung war bereits nach der Hälfte der Interviews erreicht [6].

\section{Erhebungsmethode}

Die Befragung erfolgte mittels eines theoriegeleitet entwickelten und vorab pilo- tierten Interviewleitfadens mit erzählstimulierenden Fragen. Die Interviews fanden vor Ort in den Pflegeheimen durch die Zweitautorin (diplomierte Gesundheits- und Krankenpflegerin mit Ausbildung in qualitativen Forschungsmethoden im Zuge von Lehrveranstaltungen der Erstautorin) in ungestörter Atmosphäre statt, teilweise im Rahmen der Bachelorarbeit der Zweitautorin unter Betreuung der Erstautorin und Projektleiterin. Sie wurden auf Tonband aufgezeichnet und wörtlich transkribiert. Gesprächsnotizen wurden nach jedem Interview angefertigt.

\section{Auswertungsmethode}

Die Auswertung erfolgte mittels qualitativer Inhaltsanalyse nach Mayring [16], wobei im Sinne deduktiver Kategorienanwendung eine skalierende Strukturierung nach der Ausprägung von MD (Fragestellung 1) sowie eine inhaltliche Strukturierung des Materials nach der Form von MD (cMD und uMD) vorgenommen wurde (Fragestellung 2). Die Kategoriensysteme zu den Fragestellungen 3, 5 und 6 wurden induktiv durch schrittweise Verallgemeinerung aus dem Material heraus entwickelt. Eine Kombination deduktiver und induktiver Techniken kam zur Beantwortung von Fragestellung 4 zum Einsatz. Die Auswertung erfolgte unabhängig durch beide Autorinnen im Programm MAXQDA 11, wobei zunächst die ersten 8 Interviews analysiert wurden, das Kategoriensystem sodann durch die Erstautorin überarbeitet und in mehreren Rücklaufschleifen auf das gesamte Material angewendet wurde. Nichtübereinstimmungen wurden diskutiert, bis Einigung erzielt werden konnte. Kodierregeln, Definitionen und Ankerbeispiele wurden in einem Kodierleitfaden in Form von Codememos in MAXQDA festgehalten, und ein Ablaufmodell der Analyse wurde entwickelt. Die intersubjektive Nachvollziehbarkeit als zentrales Gütekriterium qualitativer Forschung wurde durch die exakte Dokumentation des Forschungsprozesses, die Anwendung eines kodifizierten Verfahrens und das Kodieren im Team sichergestellt [25]. Die Kategoriensysteme werden un- ter Angabe der Nennungshäufigkeiten im Ergebnisteil dargestellt, zumal die qualitative Inhaltsanalyse durch ebendiese quantifizierenden Schritte „im (wenig produktiven) Streit um qualitative oder quantitative Methoden eine gewisse Zwischenstellung" einnimmt [17, S. 602].

\section{Ergebnisse}

Die Ergebnisse sind nachfolgend getrennt für die einzelnen Fragestellungen dargestellt.

\section{Ausprägung von „moral distress"}

Jedes Interview wurde deduktiv in Anlehnung an die Moral Distress Scale [1, 12, 21] hinsichtlich Intensität und Frequenz des Erlebens von moralischem Stress analysiert. Das Ergebnis dieses skalierenden Analyseschritts zeigt • Tab. 1. Der Großteil der Befragten erlebte moralischen Stress in mäßiger Ausprägung.

\section{Formen von „moral distress"}

Die Beschreibungen von MD wurden inhaltlich nach der Form von MD strukturiert. Unter cMD wurden jene Erfahrungen subsumiert, in denen die Pflegeperson eine klare Vorstellung von der moralisch korrekten Handlungsweise hatte, durch interne oder externe Barrieren jedoch an der Umsetzung gehindert wurde. Unter uMD wurden Schilderungen von Unsicherheit hinsichtlich der moralisch korrekten Handlungsweise subsumiert. Zunächst werden die Erfahrungen zu cMD näher betrachtet.

\section{In welchen Situationen wird „constraint moral distress" erlebt?}

Das induktive Kategoriensystem zu den cMD-Situationen umfasst 4 Kategorien, wobei 20 von 21 Befragten mindestens eine und maximal 3 Kategorien nannten. Wie - Tab. 2 zeigt, wurde cMD am häufigsten im Zusammenhang mit unnötigen Therapien am Lebensende erlebt, wenn Bewohner nicht sterben dürfen und durch Maßnahmen wie künstliche Ernährung oder Reanimation Leid verlängert wird. Einen Spezialfall unnöti- 
Z Gerontol Geriat 2019·52:743-750 https://doi.org/10.1007/s00391-019-01645-w

(c) Der/die Autor(en) 2019

\section{O. Kada $\cdot$ T. Lesnik}

\section{Facetten von „moral distress“ im Pflegeheim. Eine qualitative Studie mit examinierten Pflegekräften}

\section{Zusammenfassung}

Obwohl das Setting Pflegeheim einige Besonderheiten aufweist, die im Hinblick auf die Entstehung von „moral distress" (MD) bedeutsam sind, wurde MD bislang primär im Akutbereich untersucht. Die dabei zugrunde liegende klassische Definition von MD wird zunehmend kritisiert und die Erweiterung um Stress durch moralische Unsicherheit gefordert. In der vorliegenden qualitativen Studie wurde daher explorieret, in welcher Form MD von Pflegekräften in Pflegeheimen erlebt wird.

Es wurden Leitfadeninterviews mit 21 Pflegekräften aus 5 Pflegeheimen geführt. Die vollständig transkribierten Interviews wurden mittels qualitativer Inhaltsanalyse nach Mayring ausgewertet, unter Anwendung deduktiver (skalierende und inhaltliche Strukturierung) und induktiver Techniken. Ein skalierender Analyseschritt ergab, dass MD zumeist in mäßiger Ausprägung erlebt wurde. Die Befragten schilderten Situationen, in denen sie moralischen Stress erlebten, da sie durch Barrieren am moralisch korrekten Handeln gehindert worden waren. Diese Situationen ließen sich induktiv in die Kategorien „unnötige/falsche Therapie am Lebensende", "falscher Umgang mit herausforderndem Verhalten“, , schlechte Pflege allgemein" und "unnötige Krankenhaustransporte" zusammenfassen. Entscheidungen durch Angehörige und Ärzte zählten zu den meist genannten externen Barrieren; mangelnde Courage war die meist genannte interne Barriere. Die Befragten schilderten jedoch auch Stressreaktionen der Kategorie "moralische Unsicherheit", insbesondere in der "end of life care" und be Krankenhaustransportentscheidungen, v. a., wenn der Wille des Bewohners nicht bekannt war, oder aufgrund mangelnder ärztlicher Erreichbarkeit.

Die Befunde unterstreichen die Notwendigkeit einer breiten Konzeption von MD auf theoretischer und Interventionsebene.

Schlüsselwörter

Ethische Herausforderungen · Psychischer Stress $\cdot$ Ethik $\cdot$ Stress $\cdot$ Qualitative Inhaltsanalyse

\section{Facets of moral distress in nusing homes. A qualitative study with examined registered nurses}

\section{Abstract}

Although the nursing home setting has some characteristic features that are relevant with respect to the development of moral distress (MD), it has so far been studied primarily in the acute care setting. The underlying classical definition of MD is increasingly criticized and broadening of this definition is encouraged to include stress due to moral uncertainty. Hence, the present qualitative study explored how nursing personnel in nursing homes experience MD. Guided interviews were conducted with 21 nurses from 5 nursing homes. The fully transcribed interviews were analyzed using Mayring's qualitative content analysis, whereby deductive (scaling and content structuring) and inductive techniques were applied. The application of scaling structuring revealed that MD was experienced mainly in a moderately severe form. The respondents reported situations where they experienced moral stress because they were impeded by barrieres to take the morally correct action. These situations were inductively summarized in the categories "unnecessary/false treatment at the end of life", "incorrect management of challenging behavior", "poor care in general" and "unnecessary hospital transfers". Decisions made by relatives and physicians were the most frequently mentioned external barrier and a lack of courage was the most frequently named internal barrier; however, the respondents also mentioned stress reactions corresponding to the category "moral uncertainty", especially during end of life care and for hospital transfer decisions, most of all when the resident's wish was not clear or due to unavailability of physicians. The results underline the necessity of a broad conception of MD at the theoretical and interventional levels.

\section{Keywords}

Ethical issues - Psychological distress · Ethics Stress · Qualitative content analysis ger Therapien stellen Krankenhaustransporte (am Lebensende) dar. Wenn Bewohner nicht in der gewohnten Umgebung versterben dürfen und ins Krankenhaus transportiert werden, obwohl laut Ansicht der Pflegeperson kein Nutzen für den Bewohner zu erwarten ist, dann wird cMD erlebt. Außerdem wurde cMD bei unprofessionellem Umgang mit herausforderndem Verhalten von Bewohnern geschildert sowie beim Durchführen oder Beobachten ,schlechter Pflege“.

\section{Barrieren für moralisches korrektes} Handeln

Barrieren für moralisch korrektes Handeln wurden induktiv aus dem Material heraus entwickelt und deduktiv in die 2 Hauptkategorien „externe“ und „interne" Barrieren zusammengefasst.

\section{Externe Barrieren}

Alle Befragten nannten mindestens eine maximal 4 externe Barrieren für moralisch korrektes Handeln. Die meistgenannte externe Barriere waren Entschei- dungen durch Angehörige bzw. Sachwalter $(n=19)$ :

Mir geht es auch darum, wenn Bewohner sich nicht mehr äußern können, wie und wer entscheidet dann? Da gibt es oft Angehörige, die dann unbedingt wollen, dass der Bewohner künstlich ernährt wird; das ist für mich schon ein ethisch und moralisch kritischer Punkt (I15, Abs. 12; Berufserfahrung 7 Jahre, alle im Pflegeheim).

Entscheidungen bzw. Anweisungen durch Ärzte oder andere „healthcare 


\begin{tabular}{|c|c|c|c|}
\hline Kategorie & Kodierregeln (i. A. an $[1,12,21])$ & Ankerbeispiele & $n$ \\
\hline MD gering & $\begin{array}{l}\text { Eine geringe Ausprägung liegt vor, wenn } \\
\text { a) kein MD erlebt wurde (Intensität fällt } \\
\text { dann weg) oder b) MD selten und mit } \\
\text { geringer Intensität erlebt wurde }\end{array}$ & $\begin{array}{l}\text { "Das ist aber, glaube ich, auch viel Einstellungssache. Das hat ja auch } \\
\text { mit Stressresistenz zu tun. Wie sehr man sich in etwas hineinsteigert. Bei } \\
\text { mir ist es so, wenn ich in der Arbeit bin, dann bin ich in der Arbeit, und ich } \\
\text { nehme das dann nicht mit nach Hause.... Wenn es wirklich eine stark } \\
\text { belastende Situation ist, dann spreche ich mit meiner Frau darüber, die } \\
\text { kommt aus dem gleichen Bereich. Ja, das schon. Kommt aber eher selten } \\
\text { vor. Ansonsten bleibt das in der Arbeit." (I18, Abs. 12, 14; Berufserfahrung } \\
11 \text { Jahre, alle im Pflegeheim) }\end{array}$ & 5 \\
\hline MD mäßig & $\begin{array}{l}\text { Eine mittlere Ausprägung liegt vor bei } \\
\text { a) hoher Frequenz und geringer In- } \\
\text { tensität, b) bei mäßiger Intensität und } \\
\text { geringer Frequenz, oder c) wenn keine } \\
\text { eindeutige Zuordnung zu gering oder } \\
\text { hoch möglich war (ggf. widersprüchliche } \\
\text { Aussagen über das Interview hinweg) }\end{array}$ & $\begin{array}{l}\text { „Ich würde sagen, es kommt monatlich vor. Ich sage, es kommt immer } \\
\text { mehr vor. Es wird ja immer mehr vom Krankenhaus in das Pflegeheim } \\
\text { verlagert. Es wird in Zukunft sicher mehr kommen... Ich versuche, es } \\
\text { nicht mit nach Hause zu nehmen, aber es gibt sicher Situationen, die man } \\
\text { mit nach Hause nimmt und dann mit sich selbst versucht, zu lösen und zu } \\
\text { reflektieren. Man hinterfragt, warum man das so gemacht hat. Aber das } \\
\text { kommt eher selten vor." (I14, Abs. 18, 29; Berufserfahrung 4,5 Jahre, alle } \\
\text { im Pflegeheim) }\end{array}$ & 11 \\
\hline MD hoch & $\begin{array}{l}\text { Eine hohe Ausprägung liegt vor a) bei } \\
\text { mäßiger bzw. hoher Frequenz und hoher } \\
\text { Intensität, oder b) wenn ein Berufsaus- } \\
\text { stieg oder -wechsel tatsächlich erwogen } \\
\text { wurde, aufgrund von MD }\end{array}$ & $\begin{array}{l}\text { „Ja, im Prinzip erlebt man solche Situationen fast in jedem Dienst.... Ja, } \\
\text { also ich habe dann wirklich Schuldgefühle gehabt, weil ich eben der Frau } \\
\text { nicht geholfen habe.... I: Ok. Und hast du aufgrund von solchen Situatio- } \\
\text { nen, die du mir jetzt genannt hast ... schon einmal daran gedacht, dass } \\
\text { du den Beruf wechseln möchtest oder überhaupt aussteigen möchtest? B: } \\
\text { Ja, das habe ich mir schon gedacht. Nicht nur einmal. sondern des Öfte- } \\
\text { ren.... Einfach, wenn, keine Ahnung, [lachend]. Wenn viele Situationen } \\
\text { zusammenkommen. Wenn ein Sturz ist daneben und im anderen Zimmer } \\
\text { ist ein Sterbefall oder wenn einfach alles zusammenkommt. Wenn einfach } \\
\text { alles zu viel wird." (I8, Abs. 16, 34, 55, 58; Berufserfahrung 8,5 Jahre, alle } \\
\text { im Pflegeheim) }\end{array}$ & 5 \\
\hline
\end{tabular}

professionals" wurden ebenfalls häufig als externe Barrieren genannt $(n=14)$ :

Wir hatten schon eine Situation im Haus, ja. Das war eine Dame, die hatte eine $\mathrm{Na}$ sensonde. Diese war aber für mich schon im Sterbeprozess. Es gab die Anordnung, sie jedoch weiterzuernähren, obwohl sie sich die Nasensonde immer wieder selbst entfernt hat. Ich hätte sie gerne so belassen, wie sie es wollte, ohne Nasensonde. Ich wollte sie nicht auf Zwang ernähren. Das war für mich schwierig, da hätte ich gerne anders gehandelt (I14, Abs. 23; Berufserfahrung 4,5 Jahre, alle im Pflegeheim).

Das Pflegepersonal reflektiert dabei durchaus das Entscheidungsdilemma der Ärzteschaft:

Vor allem dann durch Ärzte, wenn Ärzte ebenfalls in einer Zwickmühle sind, zwischen ihrem Eid, den sie abgelegt haben, und der Situation, in welcher sie sich dann befinden. Oft sind auch Ärzte dafür verantwortlich, dass solche Stresssituationen überhaupt entstehen, weil sie dann auf
Biegen und Brechen gewisse Sachen veranlassen (I2, Abs. 26; Berufserfahrung 27 Jahre, davon 14 im Pflegeheim).

Unter „Vorgaben und Routinen“ $(n=9)$ wurden einerseits Vorgaben vonseiten des Heims kodiert:

Dass viele Bewohner um eine bestimmte Uhrzeit schlafen gehen müssen, obwohl sie vielleicht noch gerne länger aufbleiben würden. Aber um dem Nachtdienst eben etwas Arbeit abzunehmen, müssen sie schon am späten Nachmittag ins Bett (I8, Abs. 14; Berufserfahrung 8,5 Jahre, alle im Pflegeheim).

Andererseits wurde auch die Gesetzeslage als Barriere beschrieben:

Wir hatten eine Person, die war 104 Jahre alt. Sie ist dann plötzlich verstorben; diese Dame wurde dann reanimiert. Das habe ich nicht verstanden und verstehe das immer noch nicht. Das verstehe ich vom Gesetz her nicht. Ich habe noch mit der Rettung gesprochen; die haben gesagt, sie müssen sich auch an das Gesetz halten. Ich habe das nicht verstanden. Sie war bettlägerig und sehr alt; sie hatte auch keine Lebensqualität mehr. Das war ein Hammer (I15, Abs. 59; Berufserfahrung 7 Jahre, alle im Pflegeheim).

Die Kategorie Ressourcenmangel ( $n=9$ ) umfasst insbesondere Zeitmangel und fehlende Hilfsmittel als Barrieren:

Wenn diese Situationen auftreten, dann ist meistens zu wenig Personal hier. Man hat zu wenig Personal, dass man sich dann dezidiert darum kümmern kann. Dann hat man auch permanent ein schlechtes Gewissen (I6, Abs. 21; Berufserfahrung 21 Jahre, davon 18 im Pflegeheim).

Die Inkompetenz von Kollegen wurde von 6 Befragten als Barriere benannt:

Wenn der Kollege schon mit einer Abwehrspannung zu ihm geht, dann merkt der Bewohner das. Er verhält sich dann anders. Ich hatte dieses Problem nie.... Die Leute spüren das ja. Deshalb versuche ich, ihm sanfter zu begegnen und zu informieren. Es ist ja normal, dass er sich beim 


\begin{tabular}{|c|c|c|c|}
\hline Kategorie & Definitionen & Ankerbeispiele & $n$ \\
\hline $\begin{array}{l}\text { Unnötige/falsche Therapie am } \\
\text { Lebensende }\end{array}$ & $\begin{array}{l}\text { Die Pflegekraft erlebt MD durch das Beob- } \\
\text { achten oder Ausführen unnötiger Therapien, } \\
\text { die ihrer Ansicht nach nicht im besten Inte- } \\
\text { resse des Bewohners sind und nicht dessen } \\
\text { Wünschen entsprechen bzw. Leid verursa- } \\
\text { chen }\end{array}$ & $\begin{array}{l}\text { "Er [der Bewohner] bekam vor mir einen Herzstillstand } \\
\text { und er musste reanimiert werden. Ich muss schon sagen, } \\
\text { in diesem Fall finde ich eine Reanimation ethisch äußerst } \\
\text { fragwürdig. Mir kommt vor, dass viele Leute nicht mehr in } \\
\text { Frieden sterben können. Denn oft wird nicht an die Kon- } \\
\text { sequenzen, die dieser Mensch durch eine Reanimation } \\
\text { erleiden könnte, gedacht." (I6, Abs. 122; Berufserfahrung } \\
21 \text { Jahre, davon } 18 \mathrm{im} \text { Pflegeheim) }\end{array}$ & 16 \\
\hline $\begin{array}{l}\text { Falscher Umgang mit herausfor- } \\
\text { derndem Verhalten }\end{array}$ & $\begin{array}{l}\text { Die Pflegekraft erlebt MD durch das Beob- } \\
\text { achten eines ihrer Ansicht nach falschen } \\
\text { Umgangs mit Bewohnern mit herausfor- } \\
\text { derndem Verhalten }\end{array}$ & $\begin{array}{l}\text { „Wenn Heimbewohner Sedativa einnehmen müssen, um } \\
\text { ihr aggressives Verhalten zu unterdrücken bzw. den Heim- } \\
\text { bewohner zugänglicher zu machen, obwohl man in vielen } \\
\text { Fällen mit, ähm, z. B. ruhiger Stimme oder mit einer ruhi- } \\
\text { gen Ausstrahlung denselben Effekt erreichen würde." (I7, } \\
\text { Abs. 14; Berufserfahrung } 12 \text { Jahre, alle im Pflegeheim) }\end{array}$ & 11 \\
\hline Schlechte Pflege allgemein & $\begin{array}{l}\text { Die Pflegekraft erlebt MD durch das Beob- } \\
\text { achten oder Durchführen schlechter Pflege } \\
\text { (z.B. „ins Bett pflegen“, zu wenig Zuwen- } \\
\text { dung) oder nichtessenzieller pflegerischer } \\
\text { Handlungen gegen den Willen des Heimbe- } \\
\text { wohners }\end{array}$ & $\begin{array}{l}\text { „Ähm, ein Bewohner, der teilweise inkontinent ist ... und } \\
\text { der eben öfters auf das WC muss, aber es funktioniert nicht } \\
\text { mehr so. Dann habe ich schon erlebt, dass ein paar Pfle- } \\
\text { gekräfte einfach, dann wird einfach, äh, eine Inkohose } \\
\text { [Inkontinenzhose] angelegt, und der Bewohner darf dann } \\
\text { nicht mehr auf das WC, weil es einfach auch zu aufwendig } \\
\text { ist für das Pflegepersonal." (I8, Abs. } 24 \text { Berufserfahrung } \\
\text { 8,5 Jahre, alle im Pflegeheim) }\end{array}$ & 9 \\
\hline $\begin{array}{l}\text { Unnötige Krankenhaustranspor- } \\
\text { te }\end{array}$ & $\begin{array}{l}\text { Die Pflegekraft erlebt MD infolge der (bei- } \\
\text { nahen) Durchführung eines ihrer Ansicht } \\
\text { nach unnötigen, für den Bewohner belasten- } \\
\text { den Krankenhaustransports, veranlasst auf } \\
\text { Wunsch von Angehörigen oder auf ärztliche } \\
\text { Anweisung }\end{array}$ & $\begin{array}{l}\text { "Wenn ich weiß, dass es keine Hilfe mehr gibt und derjenige } \\
\text { [der Bewohner] eigentlich schon auf seinem letzten Weg ist } \\
\text { und ich ihn eigentlich nur noch plage damit, dass ich ihn ins } \\
\text { Krankenhaus schicke. Es ist... sicher für jeden belastend im } \\
\text { Krankenhaus, in der Notfallaufnahme; wissen wir alle, wie } \\
\text { das ist. Und warum kann ein Mensch nicht dort sterben, wo } \\
\text { er eigentlich zum Schluss daheim war, in seiner gewohnten } \\
\text { Umgebung?" (I3, Abs. 15; Berufserfahrung } 20 \text { Jahre, alle } \\
\text { im Pflegeheim) }\end{array}$ & 8 \\
\hline
\end{tabular}

groben Anpacken wehrt. Das löst dann eine Kettenreaktion aus, und am Ende ist dann der Bewohner der, der aggressiv ist (I16, 23-25; Berufserfahrung 15 Jahre, davon 10 im Pflegeheim).

\section{Interne Barrieren}

Insgesamt 15 von 21 Personen haben mindestens eine und maximal 2 interne Barrieren genannt. Mangelnde Courage $(n=9)$ war die meistgenannte interne Barriere:

Man denkt immer an die Situation, in welcher er [der Bewohner] sagte: ,Bitte lass mich hier im Heim. 'Ich hätte da mehr Nachdruck bei den Angehörigen leisten müssen, für den Heimbewohner. Die Angehörigen waren halt sehr dominant und grob. Ich hätte mich ihr aber trotzdem mehr stellen sollen. Über solche
Sachen denkt man schon oft nach (I9, Abs. 51; Berufserfahrung 6 Jahre, alle im Pflegeheim).

Sieben Pflegepersonen beschrieben, dass aufgrund von Überforderung und/oder negativen Gefühlen nicht moralisch korrekt gehandelt wurde:

I: Und für dich und deine Kollegen, was ist da ethisch und moralisch belastend in so einer Situation? B: Ähm, ja, wenn die Bewohnerin so ausfällig wird. Wenn sie ein bewusst aggressives Verhalten aufweist. Man ist jetzt auch als Pflegeperson nicht immer gleich gut gelaunt. Auch wenn man tagtäglich damit zu tun hat (I4, Abs. 31; Berufserfahrung 7 Jahre, davon 5 im Pflegeheim).

Zwei Pflegepersonen beschrieben außerdem eigene Wissens- bzw. Kompetenzdefizite als Barrieren für moralisch korrektes Handeln.
In welchen Situationen wird "uncertainty moral distress" erlebt?

Von 21 Befragten nannten 18 mindestens eine und maximal 3 konkrete Situationen, in denen sie uMD erlebt haben (• Tab. 3). Am häufigsten wurden Unsicherheiten in der "end of life care" als moralisch belastend beschrieben. „Uncertainty moral distress" resultierte zudem häufig im $\mathrm{Zu}$ sammenhang mit Unsicherheit darüber, ob ein Bewohner ins Krankenhaus eingewiesen werden soll oder nicht. Hierbei kam teilweise auch eine defensive Entscheidungsfindung zum Ausdruck:

Im Pflegeheim ist es viel schlimmer, du bist oft alleine im Dienst und hast niemanden zum Fragen. Wo ich dann selbst entscheiden muss, Krankenhauseinweisungen, ja oder nein. Im Zweifel weise ich lieber einmal zu viel als zu wenig ein (I12, Abs. 32; 


\begin{tabular}{|c|c|c|c|}
\hline Kategorie & Definition & Ankerbeispiel & $n$ \\
\hline End of life care & $\begin{array}{l}\text { Die Pflegekraft erlebt MD aufgrund von } \\
\text { Unsicherheiten in der end of life care. } \\
\text { Hierzu kann Unsicherheit zählen, wel- } \\
\text { che pflegerischen oder medizinischen } \\
\text { Maßnahmen am besten wären oder aber } \\
\text { Zweifel im Nachhinein, ob man es nicht } \\
\text { hätte besser/anders machen sollen }\end{array}$ & $\begin{array}{l}\text { "Der Großteil unserer Bewohner leidet an einer Demenzerkran- } \\
\text { kung, und die verschlechtert sich mit der Zeit. Das häufigste The- } \\
\text { ma bei uns ist, dass der Demenzkranke dann irgendwann aufhört } \\
\text { zu Essen. Da stellt sich dann die Frage, was wir weitermachen. } \\
\text { Viele unserer Bewohner haben noch keine Patientenverfügungen } \\
\text { oder Ähnliches, und dann liegt es oft an uns, mit den Ärzten und } \\
\text { den Angehörigen die beste Lösung für den Bewohner zu finden. } \\
\text { Nur, was ist die beste Lösung?" (I13, Abs. 14; Berufserfahrung } \\
7 \text { Jahre, davon } 4 \text { im Pflegeheim) }\end{array}$ & 12 \\
\hline $\begin{array}{l}\text { Krankenhaustransporte (am } \\
\text { Lebensende) }\end{array}$ & $\begin{array}{l}\text { Die Pflegekraft erlebt MD aufgrund von } \\
\text { Unsicherheiten, ob ein Bewohner ins } \\
\text { Krankenhaus eingewiesen werden soll } \\
\text { oder nicht. Unter diese Kategorie fallen } \\
\text { auch Zweifel an der Entscheidung zum } \\
\text { Krankenhaustransport im Nachhinein }\end{array}$ & $\begin{array}{l}\text { "Oft weiß man nicht, ab wann man jemanden in das Kranken- } \\
\text { haus schickt, oder auch der Arzt selbst ist sich auch unsicher, ab } \\
\text { und zu." (I19, Ab. 18; Berufserfahrung } 21 \text { Jahre, davon } 18 \text { im } \\
\text { Pflegeheim) }\end{array}$ & 10 \\
\hline Sonstige Situationen & $\begin{array}{l}\text { Die Pflegekraft erlebt MD infolge wei- } \\
\text { terer Situationen der moralischen Un- } \\
\text { sicherheit, die nicht unter UMD_1 oder } \\
\text { uMD_2 subsumiert werden können }\end{array}$ & $\begin{array}{l}\text { "Die freiheitseinschränkenden Maßnahmen sind auch so ei- } \\
\text { ne zwiespältige Sache. Auf der einen Seite wissen wir, dass, } \\
\text { ähm,... es in Ordnung ist, wenn wir ihm [dem Bewohner] eine } \\
\text { Sitzhose im Rollstuhl anlegen, damit er nicht aufstehen und stür- } \\
\text { zen kann, aber auf der anderen Seite möchte er das nicht und } \\
\text { wehrt sich dagegen. Aus gesetzlicher Sicht und auch von unserer } \\
\text { Vernunft her müssten wir das machen. Das sind Situationen, wo } \\
\text { man dann auch immer an die Grenzen kommt.... Gefühlsmäßig } \\
\text { ist es für mich nicht richtig jemanden festzuschnallen, der das } \\
\text { nicht will. Das ist für mich moralisch und ethisch sehr schwierig." } \\
\text { (I18, Abs. 34-37; Berufserfahrung } 11 \text { Jahre, alle im Pflegeheim) }\end{array}$ & 4 \\
\hline $\begin{array}{l}n \text { Nennungen auf Personenebene } \\
M D_{\text {"moral distress" }}\end{array}$ & & & \\
\hline
\end{tabular}

Berufserfahrung 30 Jahre, davon 5 im Pflegeheim).

Unter der Kategorie „sonstige Situationen“ wurden Schilderungen von uMD im Zusammenhang mit freiheitsbeschränkenden Maßnahmen, dem Suizid eines Bewohners, dem Tod junger Bewohner und Bewohnern, die unfreiwillig im Heim sind, subsumiert.

\section{Quellen von „uncertainty moral distress"}

Insgesamt nannten 16 der 21 Befragten mindestens eine und maximal 4 Kategorien. Moralischer Stress aufgrund von Unsicherheit kann v.a. dann entstehen, wenn der Wille des Bewohners bzw. Patienten nicht bekannt ist $(n=9)$ :

Ich finde, wenn es am Lebensende ist und es dann nicht ausgesprochen ist und ich ihn [den Bewohner] dann in das Krankenhaus schicken muss. Wenn das nicht geklärt ist. Wenn ich ihn dann noch hineinschicken muss und das eigentlich gar nichts bringt.... Ich muss es machen, aber mir selbst geht es dabei nicht gut I11, Abs. $20+22$; Berufserfahrung 7 Jahre, davon 3,5 im Pflegeheim.

Die fehlende ärztliche Erreichbarkeit im Pflegeheim ist ebenfalls eine häufig genannte Quelle von uCM $(n=9)$ :

Das ist immer eine Gratwanderung. Im Krankenhaus hat man immer mehrere Meinungen und immer die Ärzte daneben. Bei uns muss man immer alleine entscheiden. Da überlegt man schon oft, ob man das richtig entschieden hat oder ob man das anders entscheiden hätte sollen I19, Abs. 34-35; Berufserfahrung 21 Jahre, davon 18 im Pflegeheim.

Vier Pflegepersonen beschrieben Wissensmangel als eine Quelle von uMD. Pflegepersonen erleben moralischen Stress auch infolge ungelöster Konflikte und aufgrund von Schuldgefühlen im Nachhinein, wenn dem Bewohner falsche Hoffnungen bzw. Versprechungen gemacht worden waren (je $n=3$ ).

\section{Ressourcen}

Alle Befragten nannten mindestens eine und maximal 6 vorhandene Ressourcen zur Vermeidung oder Bewältigung von MD, und 16 Personen nannten mindestens eine und maximal 3 potenzielle Ressourcen. Das Pflegeteam war mit 20 Nennungen die meistgenannte vorhandene Ressource. Ärzte und Vertreter anderer Gesundheitsberufe wie z. B. Psychologen oder das mobile Palliativteam sind eine weitere wesentliche vorhandene Ressource $(n=18)$. Ebenso sind die Bewohner selbst bzw. deren Angehörige $(n=12)$ eine wichtige Ressource, wie folgendes Zitat verdeutlicht:

Die positiven Rückmeldungen von den Angehörigen und auch sehr oft von den Bewohnern, die es noch können. Und das bestärkt einen und gibt einem so viel Kraft. $\mathrm{Da}$ weiß man, man ist doch auf dem richtigen Weg I3, Abs. 58; Berufserfahrung 20 Jahre, alle im Pflegeheim.

Die Berufserfahrung nannten 16 Personen als wichtig im Umgang mit MD. 
Zwei Personen nannten Advance Care Planning (ACP) als bereits vorhandene Ressource; weitere 9 wünschten sich diese Ressource. Fort- und Weiterbildungen wurden ebenfalls als potenzielle $(n=7)$ und vorhandene $(n=1)$ Ressource genannt. Eine Teilzeitbeschäftigung beschrieben 2 Personen als bereits vorhandene Ressource, während sich 6 Personen einen erhöhten Personalschlüssel wünschten; weitere 4 Personen plädierten für Ethikcafés. Eine Person wünschte sich mehr Unterstützung durch die Führungskraft im Umgang mit MD.

\section{Diskussion}

Die vorliegende explorative Studie wurde mit der Zielsetzung durchgeführt, das Phänomen MD im Setting Pflegeheim zu explorieren in seinen verschiedenen Facetten zu beschreiben. Die Befunde der vorliegenden Studie sprechen dafür, dass Pflegepersonen im Pflegeheim moralischen Stress erleben, wenn sie durch Barrieren am moralisch korrekten Handeln gehindert werden, aber auch dann, wenn sie aufgrund mangelnder Informationen oder Ressourcen Unsicherheit hinsichtlich der moralisch korrekten Handlungsweise empfinden. Es wurde eine Vielzahl an MD-Situationen beschrieben, von Fragen der Therapiebegrenzung in der end of life care über den Umgang mit herausforderndem Verhalten bis hin zu Krankenhaustransportentscheidungen. Die Analyse von Ressourcen im Umgang mit MD unterstreicht die Bedeutung institutioneller Rahmenbedingungen, um $\mathrm{zu}$ verhindern, dass aus moralischer Spannung moralischer Stress entsteht [2].

Die Befunde der vorliegenden Studie aus dem Setting Pflegeheim unterstützen die Forderung nach einer breiten Definition von MD [5, 18]. Das Miterleben von Leid durch unnötige Therapien am Lebensende sowie unnötige Krankenhaustransporte zählten übereinstimmend mit anderen Untersuchungen im geriatrischen Kontext [20, 26] zu den häufigsten Auslösern von cMD. Das Beobachten eines unprofessionellen Umgangs mit herausforderndem Verhalten sowie das Beobachten oder Durchführen schlechter Pflege wurden als weitere Aus- löser identifiziert [2, 21]. Diesen Situationen ist gemein, dass die Pflegekraft eine klare Vorstellung über die moralisch korrekte Handlungsweise hat, diese jedoch aufgrund von Barrieren nicht umsetzen kann $[4,18]$. Entscheidungen durch Angehörige und Anweisungen von ärztlicher Seite waren hierbei die häufigsten externen Barrieren [24, 26], neben Vorgaben und Routinen des Heims und gesetzlichen Regelungen, Ressourcenmangel $[22,24]$ und Inkompetenz von Kollegen [22]. Als häufigste interne Barriere wurde mangelnde Courage [26] identifiziert, aber auch Überforderung sowie eigene Wissens- und Kompetenzdefizite [22]. In der vorliegenden Studie schilderten zahlreiche Befragte außerdem Erfahrungen von moralischem Stress durch Unsicherheit. Dies unterstreicht dieSinnhaftigkeit der Ausweitung des Konzepts MD auf Situationen moralischer Unsicherheit, Dilemmata und Konflikte, wie sie von einigen Autoren gefordert wurde $[5,18]$. Unsicherheit in der end of life care und bei Krankenhaustransportentscheidungen (am Lebensende) waren hierbei die meistgenannten auslösenden Situationen. Die moralische Unsicherheit resultiert insbesondere daraus, dass der Wille der Bewohner oftmals nicht bekannt bzw. dokumentiert ist und vom Bewohner in Anbetracht seiner gesundheitlichen Verfassung nicht mehr klar artikuliert werden kann [2]. So verfügt nur ein sehr geringer Teil von Pflegeheimbewohnern über eine Patientenverfügung [10], obwohl dies helfen kann, moralische Unsicherheit zu reduzieren [9, 22]. Auch die fragmentarische ärztliche Präsenz begünstige häufig die Entstehung von uMD $[22,26]$, sodass Maßnahmen wie geriatrische Konsiliardienste dringend $\mathrm{zu}$ empfehlen sind [9, 10]. Auch falsche Versprechungen gegenüber Bewohnern lösten moralischen Stress aus, was in einer Studie zu MD in der Pflege von Menschen mit Demenz sogar die häufigste MD-Situation darstellte [21].

„Moral distress“ wurde vom Großteil der Befragten in mäßiger Ausprägung erlebt, was durchaus mit Befunden quantitativer Studien korrespondiert [19]. Fünf Befragte berichteten von stark ausgeprägtem MD bis hin zu Überlegungen des Jobwechsels bzw. Berufsausstiegs [14, 19,
22], was die Notwendigkeit von Interventionen auf Verhaltens- und Verhältnisebene unterstreicht.

Die Befragten verfügen durchaus über Ressourcen im Umgang mit MD, die es zu stärken gilt. Dazu zählen insbesondere der Austausch im Pflegeteam, die Zusammenarbeit mit Ärzten, anderen Gesundheitsberufen sowie die Berufserfahrung. Darüber hinaus schöpfen die Befragten auch Kraft aus positiven Rückmeldungen von Angehörigen und Bewohnern.

Advance Care Planning als Maßnahme $\mathrm{zu}$ vorausschauender Planung und gemeinsamer Entscheidungsfindung unter Einbeziehung der Bewohner und der Angehörigen erscheint ein vielversprechender Ansatz zu sein, um sowohl moralische Konflikte als auch moralische Unsicherheit zu reduzieren [27]. Ganz in diesem Sinne war ACP auch die meist gewünschte Ressource zu Vermeidung bzw. Bewältigung von MD. Interprofessionelle Ethikcafés haben sich im Kontext Pflegeheim ebenfalls als vielversprechender Ansatz zur Reduktion von MD erwiesen [15] und wurden auch von mehreren Befragten als potenziell hilfreiche Ressource genannt. Darüber hinaus wünschen sich die Befragten Fort- und Weiterbildungen und mehr personelle Ressourcen.

\section{Limitationen}

Kritisch anzuführen ist die Vorauswahl der Befragten durch die jeweilige Pflegedienstleitung, wenngleich der Zugang über Gatekeeper in der qualitativen Forschung durchaus üblich ist. Im Hinblick auf die Analyse der Ausprägung von MD lässt sich durch das querschnittliche Design nicht klären, ob eine geringe Ausprägung von MD als erfolgreiche Bewältigung, geringe moralische Kompetenz oder als Ausdruck einer Distanzierung von moralischen Aspekten der eigenen beruflichen Tätigkeit und somit als Konsequenz von $\mathrm{MD}$ zu interpretieren ist [14, 19]. Jedenfalls bedarf es weiterführender Studien, um die Entstehungsbedingungen von MD im Pflegeheim auf individueller und institutioneller Ebene näher zu beleuchten, insbesondere, da eine Überprüfung der in den prominenten MDModellen [1,4] postulierten Kausalbeziehung weitgehend aussteht [14] und diese 
Modelle auf eine enge Definition von MD rekurrieren. Die Befunde der vorliegenden Studie bilden eine erste Basis für die Weiterentwicklung der MD-Theorie im Kontext Pflegeheim und die Entwicklung eines Fragebogens zur Erfassung von MD in einer breiten Definition.

\section{Fazit für die Praxis}

- Sowohl „constraint moral distress" (cMD) als auch „uncertainty moral distress" (uMD) werden im Pflegeheim erlebt, sodass für eine breite Konzeption von moralischem Stress plädiert wird.

- Die fragmentarische ärztliche Präsenz ist einerseits Ursache für moralischen Stress bei Pflegekräften, andererseits wird eine enge Zusammenarbeit mit Ärzten als wichtige Ressource in der Bewältigung von MD erlebt. Dies verdeutlicht die Notwendigkeit von Maßnahmen zur Stärkung der ärztlichen Präsenz und interdisziplinären Zusammenarbeit im Pflegeheim, wie sie beispielsweise im Rahmen einer Projektreihe zur Versorgungsoptimierung in Kärntner Pflegeheimen entwickelt und evaluiert wurden.

- Pflegekräfte beschreiben zahlreiche Ressourcen im Umgang mit MD, die für die Entwicklung von Interventionen genutzt werden können. Besonders vielversprechend erscheinen in diesem Zusammenhang neben der Förderung der individuellen Bewältigungsfähigkeit v.a. auch Maßnahmen wie Advance Care Planning (ACP) oder interprofessionelle Ethikcafés, die zur (Weiter-)Entwicklung moralischer Kompetenz beitragen.

\section{Korrespondenzadresse}

FH-Prof. Mag. Dr. Olivia Kada

Studiengang Gesundheits- und Pflegemanagement, FH Kärnten

Hauptplatz 12, 9560 Feldkirchen i.K., Österreich o.kada@fh-kaernten.at

Funding. Open access funding provided by Carinthia University of Applied Sciences (CUAS).

\section{Einhaltung ethischer Richtlinien}

Interessenkonflikt. O. Kada und T. Lesnik geben an, dass kein Interessenkonflikt besteht.

Die Teilnahme an der Befragung erfolgte freiwillig, anonym und informiert. Alle beschriebenen Untersuchungen am Menschen oder an menschlichem Gewebe wurden mit Zustimmung der zuständigen Ethikkommission, im Einklang mit nationalem Recht sowie gemäß der Deklaration von Helsinki von 1975 (in der aktuellen, überarbeiteten Fassung) durchgeführt. Von allen beteiligten Patienten liegt eine Einverständniserklärung vor.

Open Access Dieser Artikel wird unter der Creative Commons Namensnennung 4.0 International Lizenz (http://creativecommons.org/licenses/by/4.0/deed. de) veröffentlicht, welche die Nutzung, Vervielfältigung, Bearbeitung, Verbreitung und Wiedergabe in jeglichem Medium und Format erlaubt, sofern Sie den/die ursprünglichen Autor(en) und die Quelle ordnungsgemäßnennen, einen Linkzur Creative Commons Lizenz beifügen und angeben, ob Änderungen vorgenommen wurden.

\section{Literatur}

1. Corley MC, Elswick RK, Gorman M, Clor T (2001) Development and evaluation of a moral distress scale. J Adv Nurs 33(2):250-256

2. de Veer AJ, Francke AL, Struijs A, Willems DL (2013) Determinants of moral distress in daily nursing practice: a cross sectional correlational questionnaire survey. Int J Nurs Stud 50(1):100-108

3. Döring N, Bortz J (2016) Forschungsmethoden und Evaluation in den Sozial- und Humanwissenschaften, 5. Aufl. Springer, Berlin

4. Epstein EG, Hamric AB (2009) Moral distress, mora residue, and the crescendo effect. J Clin Ethics 20(4):330-342

5. Fourie C (2017) Who is experiencing what kind of moral distress? Distinctions for moving from a narrow to a broad definition of moral distress. AMA J Ethics 19(6):578-584

6. Guest G, Bunce A, Johnson L (2006) How many interviews are enough? An experiment with data saturation and variability. Field Methods 18(1):59-82

7. Jameton A (2013) A reflection on moral distress in nursing together with a current application of the concept. J Bioeth Inq 10:297-308

8. Kada 0 (2019) Moralischer Stress in der Arbeit mit geriatrischen Patientinnen und Patienten. In: Likar R, Kada O, Pinter G et al (Hrsg) Ethische Herausforderungen des Alters. Ein interdisziplinäres, fallorientiertes Praxisbuch für Medizin, Pflege und Gesundheitsberufe. Kohlhammer, Stuttgart, S212-221

9. Kada $O$ (2019) Hospital transfers of nursing home residents. A vignette experiment on nurses decision making. J Appl Gerontol 38(4):499-509. https://doi.org/10.1177/0733464816687219

10. Kada O, Janig H, Likar R, Cernic K, Pinter G (2017) Reducing avoidable hospital transfers from nursing homes in Austria: project outline and baseline results. Gerontol Geriatr Med. https://doi. org/10.1177/2333721417696671

11. Kälvemark S, Höglund AT, Hansson MG, Westerholm P, Arnetz B (2004) Living with conflicts-ethical dilemmas and moral distress in the health care system. Soc Sci Med 58(6):1075-1084
12. Kleinknecht-Dolf $M$, Spichiger $E$, Müller $M$ Bartholomeyczik S, Spirig R (2017) Advancement of the German version of the moral distress scale for acute care nurses - a mixed methods study. Nurs Open 4(4):251-266. https://doi.org/10.1002/ nop2.91

13. Laging B, Ford R, Bauer M, Nay R (2015) A metasynthesis of factors influencing nursing home staff decisions to transfer residents to hospital. J Adv Nurs 71:2224-2236. https://doi.org/10.1111/jan. 12652

14. Lamiani G, Borghi L, Argentero P (2017) When healthcare professionals cannot do the right thing: a systematic review of moral distress and its correlates. JHealth Psychol 22(1):51-67

15. Maier M, Kälin S (2016) Ethik-Cafés in der geriatrischen Langzeitpflege: halten sie, was sie versprechen?ÜberihrewahrgenommeneWirkung beim Personal und die Effekte auf verschiedene Berufsgruppen. Ethik Med 28:43-55

16. Mayring P (2015) Qualitative Inhaltsanalyse. Grundlagen und Techniken, 12. Aufl. Beltz, Weinheim

17. Mayring P (2010) Qualitative Inhaltsanalyse. In: Mey G, Mruck K (Hrsg) Handbuch qualitative Forschung in der Psychologie, 1. Aufl. VS, Wiesbaden, S601-613

18. Morley G, Ives J, Bradbury-Jones C, Irvine F (2017) What is 'moral distress'? A narrative synthesis of the literature. Nurs Ethics. https://doi.org/10.1177/ 0969733017724354 (Epubahead of print)

19. OhY,Gastmans C (2015) Moral distress experienced by nurses: a quantitative literature review. Nurs Ethics 22(1):15-31

20. Piers RD, Van den Eynde $M$, Steeman E, Vlerick $P$, Benoit DD, Van den Noortgate NJ (2012) End-oflife care of the geriatric patient and nurses' moral distress. J Am Med Dir Assoc 13(1):80.e7-80.13. https://doi.org/10.1016/j.jamda.2010.12.014

21. Pijl-Zieber EM, Awosoga O, Spenceley S, Hagen B, Hall B, Lapins J (2018) Caring in the wake of the rising tide: moral distress in residential nursing care of people living with dementia. Dementia (London) 17(3):315-336. https://doi.org/10.1177/ 1471301216645214

22. Preshaw DH, Brazil K, McLaughlin D, Frolic A (2016) Ethical issues experienced by healthcare workers in nursing homes: literature review. Nurs Ethics 23(5):490-506

23. Schreier M (2010) Fallauswahl. In: Mey G, Mruck K (Hrsg) Handbuch qualitative Forschung in der Psychologie, 1. Aufl. VS, Wiesbaden, S238-251

24. Spenceley S, Witcher CS, Hagen B, Hall B, KardolusWilson A (2017) Sources of moral distress for nursing staff providing care to residents with dementia. Dementia (London) 16(7):815-834

25. Steinke I (2004) Gütekriterien qualitativer Forschung. In: Flick U, von Kardorff E, Steinke I (Hrsg) Qualitative Sozialforschung. Ein Handbuch. Rowohlt, Reinbek bei Hamburg, S319-331

26. Young A, Froggatt K, Brearley SG (2017) 'Powerlessness' or 'doing the right thing' - moral distress among nursing home staff caring for residents at the end of life: an interpretive descriptive study. PalliatMed 31(9):853-860

27. Wöhlke S, Wiesemann C (2016) Moral distress im Pflegealltag und seine Bedeutung für die Implementierung von Advance Care Planning. Pflegewissenschaft 18(5/6):280-287 